\title{
REDUÇÃO DE SEMENTES DO TANGOR 'MURCOTE' COM A APLICAÇÃO DE BIORREGULADORES DURANTE O FLORESCIMENTO
}

\author{
Reduction of seeds in 'Honey' orange by application of plant growth regulators during \\ reproductive stages
}

\author{
Marcio Christian Serpa Domingues ${ }^{1}$, João Domingos Rodrigues ${ }^{2}$
}

\begin{abstract}
RESUMO
O presente ensaio foi conduzido em cultivo comercial do tangor 'Murcote' e teve por objetivo avaliar a atuação dos biorreguladores 2,4-D (auxina), NAA (auxina), GA 3 (giberelina) e BA (citocinina), na redução do número de sementes, sem afetar a qualidade dos frutos cítricos. Os tratamentos foram: Testemunha; 10 e 20 mg.L. de 2,4-D; 100, 150 e 200 mg.L.-1 de NAA, 100 e 200 mg.L. de GA e 20 e $40 \mathrm{mg} . \mathrm{L}^{-1} \mathrm{de}$ BA. Verificou-se que nenhum dos reguladores vegetais influenciou na qualidade dos frutos de tangor 'murcote', sem redução de peso, tamanho e teor de sólidos solúveis totais. Já em relação ao número de sementes, nenhum dos reguladores vegetais foi efetivo na redução de sementes inviáveis, porém mostraram efeito na redução de sementes viáveis, conseqüentemente com redução do número total de sementes nos frutos, quando tratados com NAA a 100 e $200 \mathrm{mg} . \mathrm{L}^{-1}$ juntamente com GA a $100 \mathrm{mg} . \mathrm{L}^{-1}$, com redução de $30 \%$ do total de sementes.
\end{abstract}

Termos para indexação: Tangor Murcote, Citrus sp, reguladores vegetais, sementes.

\begin{abstract}
The present experiment was conducted in a commercial tangor 'Murcote' citrus grove in Pratania, São Paulo State, Brazil and had the objective to evaluate the effects of, 2,4-D (auxin), NAA (auxin), GA (gibberellin) and BA (cytokinin), on the reduction of seed number, without modifications on citrus fruit quality. The treatments sprayed were as follow: control (water); 10 and $20 \mathrm{mg} . \mathrm{L}^{-1} \mathrm{of}$ 2,4-D; 100, 150 and $200 \mathrm{mg} . \mathrm{L}^{-1}$ of NAA; 100 and $200 \mathrm{mg} . \mathrm{L}^{-1}$ of GA $; 20$ and $40 \mathrm{mg} . \mathrm{L}^{-1}$ of BA. The results showed that none of plant growth regulators influenced fruit quality, without weight reduction, diameter or ${ }^{0} \mathrm{Brix}$. In relation to seed number, none of the plant growth regulators were effective on reduction of seed number, however the reduced of viable seed number and total seed number of fruits, specially with the treatment of 100 and $200 \mathrm{mg} . \mathrm{L}^{-1}$ of NAA and $100 \mathrm{mg} . \mathrm{L}^{-1}$ of GA , that showed a reduction of $30 \%$ of total seed of tangor murcott fruits.
\end{abstract}

Index terms: Honey orange, Citrus sp, plant growth regulators, seeds.

(Recebido em 20 de setembro de 2005 e aprovado em 11 de setembro de 2006)

\section{INTRODUÇÃO}

O tangor 'Murcote'é um híbrido de maturação tardia oriundo do cruzamento entre tangerina e laranja (RODRIGUEZ, 1991) e que, após sua criação nos Estados Unidos, foi transportado para o mediterrâneo, tornandose uma das frutas de maior demanda na Europa, devido ao seu aroma, aparência e quantidade de suco. Seu maior problema no mercado é a produção de grande quantidade de sementes (COHEN et al., 1989).

Segundo Lupo et al. (1991), o híbrido tangor 'Murcote' é uma cultivar geneticamente não partenocárpica, produzindo grande número de sementes e a manipulação da polinização não seria uma técnica adequada para reduzir o número de sementes.

Apesar da ação dos fatores ambientais sobre a fenologia das plantas, outro fator bastante importante são os hormônios, que estão envolvidos na maioria dos estádios de desenvolvimento dos frutos, desde a fertilização até a senescência (BUNGER-KIBLER \& BANGERTH, 1983), e por isso, os reguladores vegetais vêm tornando-se importante ferramenta para a agricultura e atualmente, já estão sendo utilizados em mais de um milhão de hectares em todo o mundo, tanto em ambiente aberto, como em cultivo protegido, aumentando a produção das culturas e, melhorando também a qualidade de órgãos vegetais produzidos (GIANFARMA, 1995).

Geralmente, a aplicação de biorreguladores para induzir frutos partenocárpicos deve ser realizada na época do florescimento das plantas, variando antes da antese ou pós- florada (LAWES et al., 1991), onde ainda é possível interferir na formação das sementes.

O processo que envolve a indução da partenocarpia traz, muitas vezes, empecilhos que acabam por prejudicar a formação dos frutos. Frutos partenocárpicos podem

1Professor Dr. do Departamento de Ciências Agrárias da Universidade de Marília - Av. Hyginno Muzzi Filho, s/n - Jardim Universitário - $17525-000$ Marília, SP - sdomingues@hotmail.com

${ }^{2}$ Professor Dr. do Instituto de Biociências/IB - Departamento de Botucatu/UNESP Botucatu - Distrito de Rubião Júnior - Cx. P. 510 - $18618-000$ Botucatu, SP _ mingo@ibb.unesp.br 
desenvolver severa deficiência de cálcio, correlacionado ao seu baixo transporte, que depende intimamente do transporte basípeto de auxina para os frutos, relacionado a força do dreno, que reduz este movimento do cálcio entre fruto-planta. Outro processo fisiológico relacionado à partenocarpia de frutos é a maior taxa de abscisão, que envolve o balanço de auxina-etileno, pois frutos partenocárpicos, por apresentarem baixo transporte de auxina, ou mesmo menor concentração de auxina pela ausência das sementes, podem apresentar alta porcentagem de queda, em função da maior concentração de etileno (BANGERTH \& SJUT, 1978).

Segundo Davies (1995) e Viviam-Smith \& Koltunow (1999), o fator que limita o crescimento do ovário não fertilizado está relacionado com a redução endógena dos níveis hormonais, pois as sementes viáveis são responsáveis por sintetizar altos níveis hormonais como auxinas, giberelinas e citocininas, e segundo Bangerth \& Schroder (1994), a aplicação exógena de biorreguladores pode induzir autonomia de crescimento do ovário das flores não fertilizadas, podendo ou não levar à formação de frutos de qualidade ou inclusive promover crescimento rápido dos frutos, continuando ou não o desenvolvimento, que vai ser influenciado pelos níveis hormonais.

A solução para a redução do número de sementes em tangor 'Murcote', não pode estar associada à manipulação da polinização das flores, pois ela é autocompatível e não partenocárpica, sendo necessário a utilização de outros métodos para a obtenção de frutos com menor número de sementes (LUPO et al., 1991).

Além disso, a forte juvenilidade das plantas oriundas de sementes faz com que sejam necessários de 10 a 15 anos para que possam ser bem avaliadas as características desejáveis como produção e qualidade dos frutos cítricos, das plantas obtidas por melhoramento vegetal (KOLLER, 1994).

Outro fator importante é a sensibilidade dos tecidos vegetais aos biorreguladores, que é influenciada pela idade dos mesmos e da presença de proteínas receptoras a estes compostos, ou seja, da concentração do complexo proteínareceptora-biorregulador; assim, a sensibilidade ao biorregulador seria simplesmente reflexo da concentração da proteína receptora (TREWAVAS, 1981, citado por ALMEIDA \& PEREIRA, 1996).

Em relação aos biorreguladores, a auxina é considerada o principal fator de crescimento dos frutos após a polinização e fertilização, e esta auxina, ou mesmo seus precursores, podem estar presentes em grãos-depólen, em óvulos e em alguns tecidos do próprio fruto
(BUCOVAC, 1963), responsáveis pelo desenvolvimento dos frutos (BALASUBRAMANANYAM \& RAGASWAMI, 1959).

Kim et al. (1994) verificaram também aumento do nível de IAA no ovário de flores de pepino, com a aplicação de inibidores de transporte de auxina (Naptalan e TIBA) no pedúnculo floral. A relação de IAA: ABA (ácido abscísico) foi maior em flores polinizadas e nas tratadas com Naftalan e TIBA (àcido 2,3,5 - triiodobenzóico) em relação às flores não polinizadas.

Segundo Talon et al. (1994), as giberelinas são responsáveis pelo desenvolvimento de frutos partenocárpicos em tangerineira 'Satsuma' e em outras cultivares de tangerineiras sem sementes, partenocarpicamente genéticas. Em tangerineira 'Ponkan', a aplicação de $\mathrm{GA}_{3}$ a $200 \mathrm{mg} . \mathrm{L}^{-1}$, promoveu redução de sementes em frutos, quando a aplicação ocorreu na antese, porém a ausência de sementes foi constatada nos menores frutos, enquanto os maiores apresentavam igual número de sementes que a testemunha. Estes resultados foram semelhantes quando a aplicação ocorreu 30 dias após a antese (TOMINAGA, 1998).

Segundo Papi \& Martinez (1984), o desenvolvimento de frutos partenocárpicos ou normais (com sementes) de tangerina 'Clementina' e também de outras espécies cítricas, é controlado pelo balanço entre $\mathrm{ABA}$ e auxina, e ABA e giberelina.

A aplicação de $\mathrm{GA}_{3}$ isolado, ou em conjunto com benziladenina (BA) ou com a auxina etilclozato (etil-cloroindazolilacetato) logo após a antese, promoveu maior pegamento de frutinhos sem sementes de pomelo, sem interferir na redução de peso dos frutos e, ainda, a combinação de $\mathrm{GA}_{3}$ mais BA na concentração de 20 e $200 \mathrm{mg} . \mathrm{L}^{-1}$, respectivamente, aumentou $\mathrm{o}^{\circ}$ Brix do suco dos frutos, em relação aos outros tratamentos e à testemunha, não ocorrendo mudanças na acidez dos frutos. Outro efeito que foi visualizado com a aplicação de $\mathrm{GA}_{3}$, BA e Ethylclozate, foi a mudança na morfologia externa dos frutos de pomelo, que demonstraram 'pescoço' próximo ao pecíolo dos frutos. A aplicação de BA, durante a divisão e nos estádios de crescimento das células, acelerou o crescimento de frutos cítricos, que apresentam auto-incompatibilidade, além de promover, juntamente com o ácido giberélico, o desenvolvimento dos frutos (NAKAJIMA et al., 1992).

A aplicação de $\mathrm{GA}_{3}$, BA e SADH (ácido 2,2dimetilhidrazida), sozinhos ou em conjunto, após a queda das pétalas, mostraram resultados variáveis, onde a mistura de $\mathrm{GA}_{3}$ e BA, aplicado dois meses após a antese, acelerou o crescimento de frutos partenocárpicos de pomelo. Este 
efeito é resultado do sinergismo existente entre o ácido giberélico e a benziladenina, pois quando aplicados isoladamente não mostraram efeito. Notou-se também que a aplicação de BA em estádios precoces de desenvolvimento, 1 mês após a antese, é mais efetivo no desenvolvimento dos frutos (SUSANTO et al., 1993).

Em Citrus sinensis, os conteúdos de ácido giberélico em genótipos de espécies e cultivares com e sem sementes mostram-se um pouco diferentes, onde as quantidades de GA são maiores em frutos partenocárpicos, como é o caso das tangerinas, devido à presença de autoincompatibilidade, apresentando mecanismo de queda principalmente nas cultivares 'clementina'. Nestas cultivares, a porcentagem de pegamento é pequena, pois não apresentam níveis adequados de ácido giberélico para o pegamento, sendo os frutos produzidos pequenos quando comparados com as 'Satsumas' (TALON et al., 1992).

Em contraste às 'Satsumas', as 'Clementinas' respondem a aplicação de GA, aumentando o pegamento dos frutos, principalmente quando aplicado entre a antese e a queda das pétalas. Para ambas as cultivares, a aplicação de paclobutrazol (inibidor de síntese de ácido giberélico) aumentou a abscisão de frutos, sendo este efeito completamente invertido pela aplicação exógena do ácido giberélico, concluindo que a redução do GA endógeno promove queda de frutos, através da oxidação do IAA causado pelo triazol, e que o aumento dos níveis de GA diminuem a abscisão (TALON et al., 1992).

Makita (1994) obteve a produção de frutos partenocárpicos do tangor 'Kyiomi', com a aplicação de GAP (Pasta de lanolina $+\mathrm{GA}_{3}$, na concentração de 2,7\%) no estigma das flores, no pedúnculo floral e na base das gemas de florescimento, mas também observou aumento na acidez dos frutos, atraso na coloração e presença de frutos pequenos e mal formados.

Em frutos cítricos, a aplicação de $\mathrm{GA}_{3}$ aumentou o pegamento de frutos em tangerineiras, onde sua aplicação no pistilo das flores resultou em melhor mobilização de metabólitos para o ovário (POWELL \& KREZDORN, 1977).

Segundo Feinstein et al. (1975), em tangerineira 'Dancy' e 'Temple', o GA 3 na dosagem de 20 mg.L - $^{-1}$ e o NAA a $150 \mathrm{mg} . \mathrm{L}^{-1}$, aplicados durante o pleno florescimento, reduziu o número de sementes em $28 \%$ em 'dancy' e $18 \%$ em 'temple', onde o efeito do NAA foi melhor que do $\mathrm{GA}_{3}$. Porém, o número de sementes inviáveis foi maior nos frutos tratados com NAA, bem como nestes tratamentos ocorreu maior redução de sementes viáveis, e as sementes inviáveis nem foram detectadas no momento da contagem das sementes (maturação fisiológica). O NAA causou o abortamento de muitos óvulos no estádio precoce de desenvolvimento dos frutos, onde sementes inviáveis nem foram detectadas no momento da contagem das sementes, e o $\mathrm{GA}_{3}$ promoveu aumento do número de frutos, porém com peso inferior dos frutos e redução de sementes viáveis e inviáveis.

Diante disso, buscou-se com este trabalho avaliar a eficiência de reguladores vegetais na indução da formação de frutos com menor número de sementes de Tangor 'Murcote' (Citrus sinensis L. Osbeck x Citrus reticulata Blanco), baseado na obtenção de frutos de mesmas características qualitativas e quantitativas.

\section{MATERIAL E MÉTODOS}

$\mathrm{O}$ experimento foi realizado em propriedade comercial de produção de Tangor 'Murcote', de 5 anos e produtividade média de 1,5 caixa de 40,8 Kg, localizado no município de Pratânia, Estado de São Paulo, nas coordenadas geográficas $22^{\circ} 44^{\prime}$ de Latitude Sul e $48^{\circ} 34^{\prime}$ de Longitude Oeste, com $700 \mathrm{~m}$ de altitude.

O clima do local do experimento é classificado, segundo KÖPPEN, como mesotérmico cfa, clima temperado chuvoso, com total de chuvas no mês mais seco menor que $40,7 \mathrm{~mm}$ e onde o mês mais quente apresenta temperatura média de $23,8^{\circ} \mathrm{C}$, e o mês mais frio $17,5^{\circ} \mathrm{C}$, com temperatura média anual de $21^{\circ} \mathrm{C}$ e precipitação média de $1445 \mathrm{~mm}$.

O experimento foi instalado no momento do florescimento, que ocorreu no mês de setembro de 1999. Foi realizada somente uma aplicação dos biorreguladores, em 15 de setembro de 1999, onde a maioria das flores estavam abertas (Figura 1).

Os tratamentos realizados foram os seguintes: 1Testemunha - água; T2-Auxina - 2,4-D - 10 mg.L.1 ; T3auxina - 2,4-D - 20 mg.L-1 ${ }^{-1}$ T4-Auxina - NAA - 100 mg.L${ }^{1}$;T5- Auxina - NAA - 150 mg.L-1;T6- Auxina - NAA - 200 mg.L.- $;$ T7- Giberelina - GA -100 mg.L - $^{-1}$;T8- Giberelina -

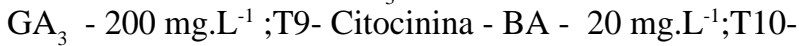
Citocinina - BA - $40 \mathrm{mg} . \mathrm{L}^{-1}$.

$\mathrm{O}$ ácido giberélico utilizado foi na formulação comercial de Pró-Gibb, que contém $10 \%$ de GA. As auxinas utilizadas foram o 2,4-D (éster butírico do ácido 2,4 diclorofenoxi-acético), de nome comercial Esteron 400 BR, que contém 400 g.L $\mathrm{L}^{-1}$ de i.a., e o NAA (ácido naftaleno acético) de nome comercial Nafusaku. A citocinina utilizada, foi a BA ( 6 - benzilaminopurina), uma citocinina purínica, produto puro.

Ciênc. agrotec., Lavras, v. 31, n. 3, p. 758-764, maio/jun., 2007 


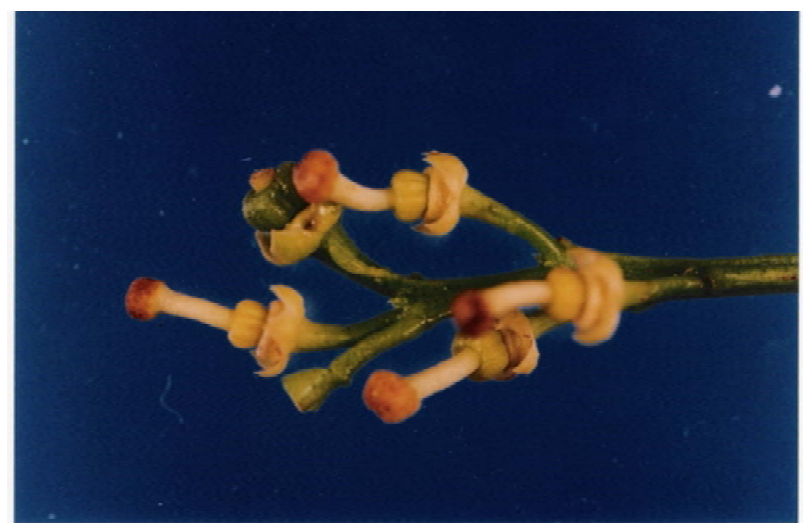

FIGURA 1 - Ramo floral com botão e ovário em desenvolvimento, sem pétalas de tangor 'Murcote' (Citrus sinensis L. Osbeck x Citrus reticulata Blanco), setembro/ 1999, Pratânia/SP.

As plantas foram pulverizadas, com 3 litros da solução por planta, acrescido de $0,05 \%$ de espalhante adesivo não iônico, do grupo químico dos alquilfenoxietoxilados, cujo ingrediente ativo é o Alquil fenol poliglicoléter, na concentração de 250 g.L.-1 , produto comercial de nome Extravon.

$\mathrm{O}$ experimento foi instalado em blocos ao acaso, com 4 repetições, contendo 2 plantas por parcela, 8 plantas por tratamento, totalizando 144 plantas, analisados estatisticamente pelo teste Duncan, ao nível de 5\% de probabilidade .

Cada planta foi dividida em 4 quadrantes, e em dois deles foram marcados aleatóriamente dois ramos (1 ramo por quadrante), na altura do peito, a cerca de 1 metro de altura, onde foram realizadas as seguintes avaliações: Porcentagem de abscisão de frutos; Número de sementes viáveis por fruto; Número de sementes inviáveis por fruto; Número total de sementes por fruto e Diâmetro dos frutos.

A classificação das sementes em viáveis e inviáveis, levou em consideração a presença ou ausência de material de reserva e embrião onde, sementes geralmente pequenas e chochas (vazias) eram classificadas como sementes inviáveis, e sementes cheias, classificadas como sementes viáveis, ou seja, aptas à germinação. Já o diâmetro dos frutos foi determinado como o auxílio de paquímetro digital, para relacionar tal parâmetro com a quantidade de sementes presentes nos frutos.

\section{RESULTADOS E DISCUSSÃO}

De acordo com o Quadro 1, que mostra os resultados de diâmetro dos frutos do tangor 'Murcote' no momento da colheita, quando os mesmos se encontravam maduros, verifica-se que nenhum dos biorreguladores estimularam maior desenvolvimento em diâmetro dos frutos.

É possível observar o efeito dos reguladores vegetais na redução do número total de sementes nos frutos de tangor 'Murcote', verificando que ocorreu o abortamento do zigoto (óvulo fecundado) através da análise visual em lupa, mesmo com a polinização acontecendo normalmente, sendo a aplicação desses reguladores vegetais, efetuada em pleno florescimento, onde alguns reguladores promoveram a redução da formação de sementes.

Os melhores tratamentos, que diferiram estatisticamente da testemunha, em relação ao número total de sementes, foram aqueles que apresentavam NAA $100 \mathrm{e}$ $200 \mathrm{mg} . \mathrm{L}^{-1}$ e GA 3 a $100 \mathrm{mg} . \mathrm{L}^{-1}$, que induziram a redução do número total de sementes (Quadro 1). NAA na concentração intermediária, também se mostrou eficiente, porém não diferiu estatisticamente da testemunha.

Estes resultados estão de acordo com Feinstein et al. (1975), que também obtiveram redução do número total de sementes em tangerineiras 'Dancy' e 'Temple', também com a aplicação de $\mathrm{GA}_{3}$ e NAA, em pleno florescimento.

Dentre os tratamentos, somente o NAA (100 mg. $\left.\mathrm{L}^{-1}\right)$ promoveu a redução do número de sementes inviáveis, contribuindo para redução do número total de sementes (Quadro 1). Argumenta-se que esta redução do número de sementes pode estar relacionada ao abortamento do embrião causado pela auxina. Outro tratamento que se mostrou efetivo para a redução de sementes inviáveis foi BA $\left(20 \mathrm{mg} . \mathrm{L}^{-1}\right)$, mas mesmo assim não contribuiu para a redução do número total de sementes, que representa o valor mais importante deste estudo.

O NAA, é uma auxina bastante conhecida como biorregulador que pode induzir a síntese de etileno, e inclusive é muito utilizada para promover a abscisão (DAVIES, 1995). As concentrações utilizadas neste experimento, 100 e $200 \mathrm{mg} . \mathrm{L}^{-1}$, não promoveram a abscisão de frutinhos de tangor 'Murcote', embora tenham promovido o crescimento dos frutos. Possivelmente este biorregulador foi eficiente no abortamento de óvulos com redução do número total de sementes, sendo que as concentrações aplicadas teoricamente mantiveram níveis adequados de auxinas para promover o crescimento dos frutos, em substituição à concentração hormonal endógena, que foi reduzida em conseqüência da diminuição do número de sementes (TALON et al., 1992). 
QUADRO 1 - Diâmetro médio (mm), número de sementes viáveis, inviáveis e número total de sementes de frutos de Tangor 'Murcote' (Citrus sinensis L. Osbeck x Citrus reticulata Blanco) na época da colheita dos frutos, junho/2000, Pratânia/SP.

\begin{tabular}{|c|c|c|c|c|}
\hline Tratamentos & $\begin{array}{l}\text { Diâmetro } \\
(\mathbf{m m})\end{array}$ & $\begin{array}{c}\text { Número de } \\
\text { sementes inviáveis }\end{array}$ & $\begin{array}{l}\text { Número de } \\
\text { sementes } \\
\text { viáveis }\end{array}$ & $\begin{array}{l}\text { Número total de } \\
\text { sementes }\end{array}$ \\
\hline 1. Testemunha & $68,42 \mathrm{ab}$ & $35,75 \quad a b c$ & 214,00 a & 250,00 a \\
\hline 2. Auxina $-2,4-\mathrm{D}-10 \mathrm{mg} . \mathrm{L}^{-1}$ & $67,66 \mathrm{ab}$ & 38,75 abc & 183,25 abc & $222,00 \mathrm{ab}$ \\
\hline 3. Auxina $-2,4-\mathrm{D}-20 \mathrm{mg} \cdot \mathrm{L}^{-1}$ & $69,57 \mathrm{a}$ & $40,50 \mathrm{abc}$ & 181,75 abc & $222,25 \mathrm{ab}$ \\
\hline 4. Auxina - NAA - $100 \mathrm{mg} . \mathrm{L}^{-1}$ & $68,33 \mathrm{ab}$ & $30,00 \mathrm{c}$ & $152,75 \mathrm{bc}$ & $180,50 \mathrm{~b}$ \\
\hline 5. Auxina - NAA - $150 \mathrm{mg} \cdot \mathrm{L}^{-1}$ & $67,15 \mathrm{ab}$ & $40,50 \mathrm{abc}$ & $165,25 \mathrm{bc}$ & $205,75 \mathrm{ab}$ \\
\hline 6. Auxina - NAA - $200 \mathrm{mg} \cdot \mathrm{L}^{-1}$ & $66,42 \mathrm{ab}$ & 39,25 abc & $158,50 \mathrm{bc}$ & $197,75 \quad b$ \\
\hline 7. Giberelina $-\mathrm{GA}_{3}-100 \mathrm{mg} \cdot \mathrm{L}^{-1}$ & $68,76 \mathrm{ab}$ & $40,00 \mathrm{abc}$ & $161,50 \mathrm{bc}$ & $199,25 \mathrm{~b}$ \\
\hline 8. Giberelina $-\mathrm{GA}_{3}-200 \mathrm{mg} . \mathrm{L}^{-1}$ & $68,56 \mathrm{ab}$ & $50,75 \mathrm{ab}$ & 169,75 abc & $220,50 \mathrm{ab}$ \\
\hline 9. Citocinina - BA - 20 mg. $\mathrm{L}^{-1}$ & $66,81 \mathrm{ab}$ & $31,50 \mathrm{bc}$ & $187,25 \mathrm{ab}$ & $218,75 \mathrm{ab}$ \\
\hline 10. Citocinina - BA - $40 \mathrm{mg} \cdot \mathrm{L}^{-1}$ & $66,60 \mathrm{ab}$ & $40,75 \mathrm{abc}$ & $165,50 \mathrm{bc}$ & $206,25 \mathrm{ab}$ \\
\hline Coeficiente de variação (\%) & 1,60 & 14,86 & 7,75 & 14,05 \\
\hline
\end{tabular}

Médias seguidas de mesma letra, não diferem entre sí na vertical, pelo teste Duncan ao nível e 5\% de probabilidade.

As auxinas, 2,4-D e NAA, que foram aplicadas sobre o tangor 'Murcote' não interferiram no tamanho dos frutos (diâmetro), parâmetro qualitativo de comercialização de frutos cítricos. O único efeito, e também o mais importante, foi a redução do número total de sementes, com a aplicação de NAA. O 2,4-D não se mostrou eficiente na redução do número de sementes.

Os resultados referentes à redução do número de sementes viáveis estão de acordo com os resultados obtidos por Feinstein et al. (1975) que relata que a aplicação de NAA na concentração de 150 e $200 \mathrm{mg} . \mathrm{L}^{-1}$ reduziu o número de sementes viáveis em frutos de tangerineira 'Dancy' e 'Temple', onde a alta concentração de NAA no ovário promoveu o abortamento dos óvulos, e o desenvolvimento.

A giberelina envolvida $\left(\mathrm{GA}_{3}\right)$ e a citocinina benzilaminopurina (40 mg. $\left.\mathrm{L}^{-1}\right)$, também induziram a redução do número de sementes viáveis e do número total de sementes nos frutos de tangor 'Murcote', sem afetar qualitativamente os frutos, em relação ao tamanho dos frutos. As giberelinas e as citocininas são promotores do metabolismo e podem induzir a redução do número de sementes em diversas espécies de plantas hortícolas (DAVIES, 1995; HOPKINS, 1999; TAIZ \& ZEIGER, 1998), principalmente naquelas espécies de planta que já são consideradas geneticamente partenocárpicas.
De acordo com os vários trabalhos científicos analisados, o $\mathrm{GA}_{3}$ aplicado com a finalidade de decréscimo do número de sementes, também mostrou efeito na redução da qualidade dos frutos. Em tangor 'Murcote', apesar do $\mathrm{GA}_{3}$ ter proporcionado redução do número de sementes, tal redução não pode ser considerada tão eficiente, apesar de ter existido. Esta eficiência pode estar relacionada com a rápida metabolização do $\mathrm{GA}_{3}$, principalmente para $\mathrm{o}$ crescimento dos frutos cítricos, pois suas taxas de crescimento são mais lentas, em relação a outros tipos de frutos, como as olerícolas, onde o ciclo reprodutivo é mais curto, e as plantas são anuais e bienais (BANGERTH \& SCHRODER, 1994).

Ainda de acordo com os mesmos autores, que injetaram $\mathrm{GA}_{3}$ marcado com ${ }^{14} \mathrm{C}$ no ovário de flores de tomateiro e macieira, obtiveram frutos bem desenvolvidos sem sementes, mas verificaram ser o $\mathrm{GA}_{3}$ completamente metabolizado em menos de 48 horas. Para o tangor 'Murcote', que apresenta desenvolvimento mais lento, seria necessário maior tempo de atuação do $\mathrm{GA}_{3}$ para promover redução mais drástica no número de sementes. Em tangerina 'Ponkan', de acordo com Tominaga (1998), o GA 3 reduziu o número de sementes, mas os frutos se tornaram menores e mais ácidos.

Segundo Powell \& Krezdorn (1977), a efetividade do $\mathrm{GA}_{3}$ pode estar relacionada também com o tipo de

Ciênc. agrotec., Lavras, v. 31, n. 3, p. 758-764, maio/jun., 2007 
aplicação, que se for mais localizada, no pistilo das flores, promove melhor mobilização dos metabólitos para o ovário e, conseqüentemente, maior pegamento dos frutos com menor número de sementes.

Talon et al. (1992) afirmaram que o efeito e redução no número de sementes e o crescimento do fruto, está diretamente relacionado com as giberelinas, pois em tangerina 'Satsuma' e outras tangerinas geneticamente partenocárpicas, elas é que são consideradas responsáveis pelo crescimento dos frutos.

\section{CONCLUSÕES}

Os reguladores vegetais, principalmente os promotores do metabolismo, podem ser utilizados para induzir a redução do número de sementes em tangor 'Murcote', o qual apresenta elevado número de sementes.

Nenhum dos reguladores vegetais aplicados influenciou a qualidade dos frutos de tangor 'Murcote', em relação ao tamanho.

O NAA nas concentrações de 100 e $200 \mathrm{mg} . \mathrm{L}^{-1}$, o $\mathrm{GA}_{3}$ na concentração de $100 \mathrm{mg} . \mathrm{L}^{-1}$,foram os tratamentos mais efetivos na redução do número total de sementes. Além disso, estes resultados sugerem a necessidade de maiores investigações a respeito da ação destes reguladores vegetais, em frutos que geneticamente não são partenocárpicos, como o tangor 'Murcote'.

\section{REFERÊNCIAS BIBLIOGRÁFICAS}

ALMEIDA, J. A. S.; PEREIRA, M. F. D. A. Efeito de GA e paclobutrazol no desenvolvimento vegetativo do girassol. Revista Brasileira de Fisiologia Vegetal, Londrina, v. 9, n. 1, p. 55-60, 1996.

BALASUBRAMANANYAM, V. R.; RANGASWAMI, G. Parthenocarpy in guava induced by pollen hormone. Current Science, Bangalore, v. 28, p. 413-415, 1959.

BANGERTH, F.; SCHRODER, M. Strong synergistic effects of gibberellins with the synthetic cytokinin N-(2-chloro-4pyridyl)-N-phenylurea on parthenocarpic fruit set and some other fruit characteristics of apples. Plant Growth Regulation, [S.1.], v. 15, p. 293-302, 1994.

BANGERTH, F.; SJUT, V. Induced parthenocarpy: a tool for investigation hormone regulated physiological processes in fruits. Acta Horticulturae, Wageningen, v. 80, p. 169-174, 1978.
BUKOVAC, M. J. Induction of parthenocarpic growth of apple fruits with gibberellins $\mathrm{A}_{3}$ e $\mathrm{A}_{4}$. Botanical Gazettee, [S.1.], n. 124, p. 191-195, 1963.

BUNGER-KLIBER, S.; BANGERTH, F. Relationship between cell number, cell size of seeded fruits of tomato (Lycopersicon esculentum Mill.) and those parthenocarpically by the application of plant growth regulators. Plant Growth Regulation, [S.1.], n. 1, p. 143154, 1983.

COHEN, A.; LABON, R.; CHISDAY, D.; YAIR, A. Decreasing of seed number in murcott and others cultivars. Alon Hanotea, [S.1.], v. 43, n. 8, p. 945-947, 1989.

DAVIES, P. J. (Ed.). Plant hormones. 2. ed. Dordrecht: Kluwer Academic, 1995. 833 p.

FEINSTEIN, B.; MONSELISE, S. P.; GOREN, R. Studies on the reduction of seed number in mandarins. Hortscience, Alexandria, v. 10, p. 385-386, 1975.

GIANFARMA, T. Natural and synthetic growth regulators and their use in horticultural and agronomic crop. In: DAVIES, P. J. (Ed.). Plant hormones. 2. ed. Dordrecht: Kluwer Academic, 1995. 833 p.

HOPKINS, W. G. Introduction to plant physiology. 2. ed. New York: J. Wiley, 1999. 512 p.

KIM, I. S.; OKUBO, H.; FUJIEDA, K. Studies on parthenocarpy in Cucumis sativus L.: IV. effects of exogenous growth regulators on induction of parthenocarpy and endogenous hormone levels in cucumber ovaries. Journal of the Korean Society for Horticultural Science, [S.1.], v. 35, p. 187-195, 1994.

KOLLER, O. C. Citricultura. Porto Alegre: Rígel, 1994. 446 p.

LAWES, G. S.; WOOLEY, D. J.; CRUZ-CASTILHO, J. G. Field responses of kiwifruit to CPPU (cytokinin) application. Acta Horticulturae, Wageningen, v. 297, 1991.

LUPO, A.; EISIKOWITCH, D.; BROSH, P. Pollination in murcott cultivar of citrus (Rutaceae), the influence on seed number and productivity. Acta Horticulturae, Wageningen, n. 288 , p. 275-277, 1991 . 
MAKITA, Y. Studies on thr production of seedless fruit from seeded Citrus types II: production of seedless fruits in Hyuganatsu and Kiyomi tangor by gibberellin paste treatment. Bulletin Shizuoka Prefecture Citrus Experimental Station, [S.1.], n. 20, p. 21-29, 1994.

NAKAJIMA, Y.; SUSANTO, S.; HASEGAWA, K. Effect of growth hormone application on the incidence of parthenocarpic and fruit quality of pummelo trees grown in plastic house. Japanese Journal of Tropical Agriculturae, [S.1.], v. 36, n. 4, p. 263-268, 1992.

PAPI, G.; MARTINEZ, G. Endogenous plant growth substance content in young fruits of seeded and seedless Clementine mandarins as related to fruit set and development. Sciencia Horticulturae, Canterbury, v. 22, p. 265-274, 1984.

POWELL, A. A.; KREZDORN, A. H. Influence of fruit setting treatment on translocation of ${ }^{14} \mathrm{C}$ metabolites in citrus during flowering and fruit. Journal of the American Society for Horticultural Science, Alexandria, v. 102, p. 709-714, 1977.

RODRIGUEZ, O. (Ed.). Citricultura brasileira. 2. ed. Campinas: Fundação Cargill, 1991. v. 1, 492 p.
SUSANTO, S.; NAKAJIMA, Y.; HASEGAWA, K. Effect of growth hormone application on the development of parthenocarpic fruits of pummelo trees growth in a plastic house. Japanese Journal of Tropical Agriculture, [S.I.], v. 37, p. 284-289, 1993.

TAIZ, L.; ZEIGER, E. Plant physiology. 2. ed. Sunderland: Sinauer Associates, 1998. 792 p.

TALON, M.; ZACARIAS, L.; PRIMO-MILLO, E. Role of gibberellins in parthenocarpic development of seedless mandarins. Proceedings of International Society of Citriculture, [S.1.], v. 1, p. 485-488, 1994.

TALON, M.; ZACARIAS, L.; PRIMO-MILLO, E. Gibberellins and parthenocarpic ability in developing ovaries of seedless mandarins. Plant Physiology, Washington, v. 99, p. 1575-1581, 1992.

TOMINAGA, S. GA sprays delay and reduce physiological fruit drop in ponkan mandarin (Citrus reticulata Blanco). Acta Horticulture, Wageningen, v. 463, p. 301-305, 1998.

VIVIAN-SMITH, A.; KOLTUNOW, A. M. Genetic analysis of growth regulator induced parthenocarpy in arabidopsis. Plant Physiology, Washington, v. 121, p. 437-451, 1999. 